\title{
INVESTIGATION OF PHONONS IN HgCdMnTe USING FAR-INFRARED REFLECTIVITY
}

\author{
E. Dudziak, J. Bożym, D. Pruchnik \\ Institute of Physics, Technical University of Wrocław \\ Wybrzeże Wyspiańskiego 27, 50-370 Wrocław, Poland \\ AND J. BARAN \\ Institute of Low Temperature and Structure Research, Polish Academy of Sciences \\ Okólna 2, 50-950 Wrocław, Poland
}

\begin{abstract}
The first reflectivity spectra of $\mathrm{Hg}_{1-x-y} \mathrm{Cd}_{x} \mathrm{Mn}_{y}$ Te with $0.03<x<0.1$ and $0<y<0.05$ were measured in the spectral region $700-30 \mathrm{~cm}^{-1}$ at $300 \mathrm{~K}$ and $90 \mathrm{~K}$. The quaternary alloys measured show three mode behaviour. The experimental results are interpreted by using a classical dynamic dielectric function model.
\end{abstract}

PACS numbers: 78.30.Fs, 71.45. Gm

\section{Introduction}

The quaternary alloy $\mathrm{Hg}_{1-x-y} \mathrm{Cd}_{x} \mathrm{Mn}_{y}$ Td is an interesting diluted magnetic semiconductor because of possibility of additional control of its material parameters. Important physical properties such as band gap, lattice constant, magnetization can be changed independently of each other by proper choosing its molar compositions $x$ and $y$ [1]. HgCdMnTe is a competitive material to the widely used IIgCdTe semiconductor for infrared detectors [2].

\section{Experiment}

$\mathrm{HgCdMnTe}$ samples (of sizes about $8 \times 8 \mathrm{~mm}^{2}$ ) used in experiment were the epitaxial layers (about $200 \mu \mathrm{m}$ thick) grown by isothermal vapour phase epitaxy in the way similar to that described in [3]. Single crystals of CdMnTe with $5-30 \%$ MnTe molar content were used as a substrate. The molar compositions were measured by electron microprobe. Four samples with molar compositions $(0.03<x<0.1$ and $0<y<0.05)$ were used in the experiment.

Reflectivity measurements at $300 \mathrm{~K}$ and $90 \mathrm{~K}$ were made with Bruker IF-88 Fourier transform spectrometer in the spectral region $700-30 \mathrm{~cm}^{-1}$. The pyroelectric TGS and Si bolometer working at $4.2 \mathrm{~K}$ were used as detectors in different spectral regions. 


\section{Results and discussion}

The far-infrared reflectivity spectra measured on exemplary sample are presented in Fig. 1. Only the most interesting spectral range of the lattice resonance $250-30 \mathrm{~cm}^{-1}$ is shown. It is clearly seen that quaternary $\mathrm{HgCdMnTe}$ mixed crystals are characterized by three well resolved reststrahlen bands near the $\mathrm{HgTe}$-, CdTeand MnTe-like TO-phonon modes. There is an additional peak or bump (called $\Omega_{2}$ mode in [7]) below the HgTe-like mode. This additional band was observed in $\mathrm{HgTe}[4,5]$ and in other $\mathrm{Hg}$ compounds and $\mathrm{Hg}$-rich mixed crystals, i.e. [6-12]. We performed a standard Kramers-Kronig analysis which yields the dielectric function $\varepsilon(\omega)$ and mode frequencies without presupposing any model for response function. The spectra of $\operatorname{Im} \varepsilon$ and $\operatorname{Im}(-1 / \varepsilon)$ were calculated and in this way the frequencies of transverse optical-phonon modes and frequencies of longitudinal excitations (coupled plasmon-longitudinal phonon modes $\omega_{\mathrm{LO}}-\omega_{\mathrm{p}}$ ) determined. These data were the start points in the fitting procedure. We used the dynamical dielectric function model applied in $[4,6]$ to describe the reflectivity spectra. In this model the
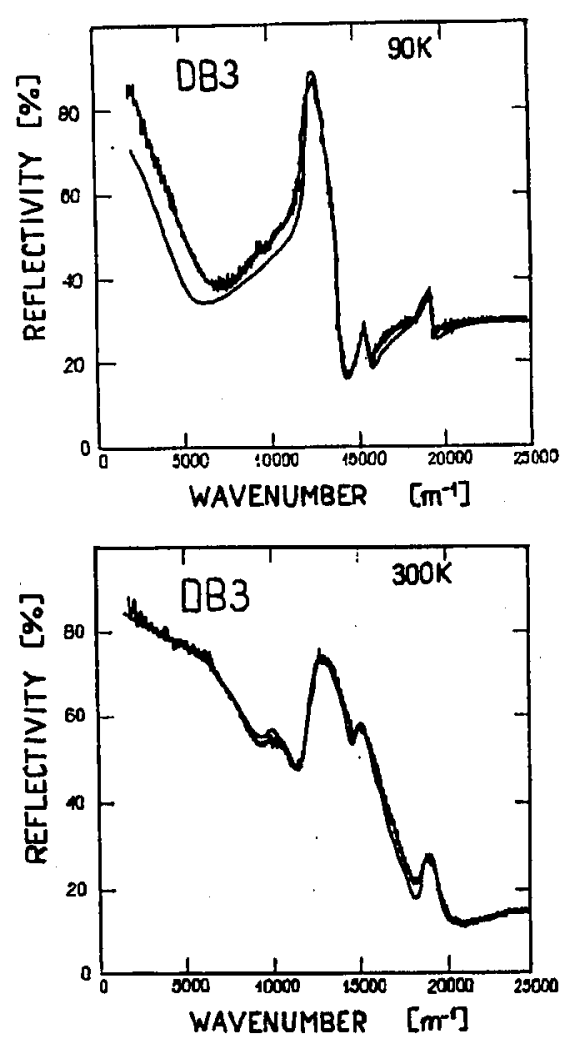

Fig. 1. Far-infrared reflectivity spectra for $\mathrm{Hg}_{1-x-y} \mathrm{Cd}_{x} \mathrm{Mn}_{y} \mathrm{Te}$ with $x=0.067$ and $y=0.03$ at $90 \mathrm{~K}$ and $300 \mathrm{~K}$ (noisy line - experimental, smooth line - calculated). 
TABLE

\begin{tabular}{|c|c|c|c|c|c|c|c|c|c|}
\hline \multirow[b]{2}{*}{ Sample } & \multicolumn{3}{|c|}{$\mathrm{MnTe}$} & \multicolumn{3}{|c|}{$\mathrm{CdTe}$} & \multicolumn{3}{|c|}{$\mathrm{HgTe}$} \\
\hline & $\begin{array}{c}\omega_{\mathrm{TO}} \\
{\left[\mathrm{cm}^{-1}\right]}\end{array}$ & $\begin{array}{c}\Gamma \\
{\left[\mathrm{cm}^{-1}\right]} \\
\end{array}$ & $S$ & $\begin{array}{c}\omega_{\mathrm{TO}} \\
{\left[\mathrm{cm}^{-1}\right]}\end{array}$ & $\begin{array}{c}\Gamma \\
{\left[\mathrm{cm}^{-1}\right]}\end{array}$ & $S$ & $\begin{array}{c}\omega_{\mathrm{TO}} \\
{\left[\mathrm{cm}^{-1}\right]}\end{array}$ & $\begin{array}{c}\Gamma \\
{\left[\mathrm{cm}^{-1}\right]}\end{array}$ & $S$ \\
\hline $\begin{array}{l}\text { DB3: } \\
300 \mathrm{~K}\end{array}$ & 187 & 7.9 & 0.11 & 148 & 6.7 & 0.10 & 122 & 12 & 4.3 \\
\hline $90 \mathrm{~K}$ & 191 & 4.5 & 0.175 & 153 & 3.35 & 0.10 & 121 & 2.0 & 4.5 \\
\hline $\begin{array}{l}\text { DA3: } \\
300 \mathrm{~K}\end{array}$ & 188 & 11 & 0.2 & 149 & 6.7 & 0.15 & 120 & 7 & 3.8 \\
\hline $90 \mathrm{~K}$ & 190 & 7 & 0.25 & 151 & 4.1 & 0.12 & 119 & 1.5 & 3.9 \\
\hline & & $\Omega_{2}$ & & & Plasmon & & & & \\
\hline Sample & $\begin{array}{c}\omega_{\mathrm{TO}} \\
{\left[\mathrm{cm}^{-1}\right]}\end{array}$ & $\begin{array}{c}\Gamma \\
{\left[\mathrm{cm}^{-1}\right]}\end{array}$ & $S$ & $\begin{array}{r}N e^{2} / \\
{\left[\times 10^{5}\right.} \\
\end{array}$ & $\begin{array}{l}E_{0} \mathrm{~m}^{*} \\
\left.\mathrm{~cm}^{-2}\right]\end{array}$ & $\begin{array}{c}\Gamma_{\mathrm{p}} \\
{\left[\mathrm{cm}^{-1}\right]}\end{array}$ & & & \\
\hline $\begin{array}{l}\text { DB3: } \\
300 \mathrm{~K}\end{array}$ & 98 & 15 & 2.9 & 2. & & 65 & & & \\
\hline $90 \mathrm{~K}$ & 105 & 25.5 & 2.0 & 0. & & 40 & & & \\
\hline DA3: & & & & & & & & & \\
\hline $300 \mathrm{~K}$ & 98 & 25 & 3.5 & 3. & & 250 & & & \\
\hline $90 \mathrm{~K}$ & 100 & 50 & 2.5 & 7. & & 400 & & & \\
\hline
\end{tabular}

dielectric function is of the form

$$
\varepsilon(\omega)=\varepsilon_{\infty}^{\prime}+\Delta \varepsilon_{\text {inter }}(\omega)-\frac{N e^{2}}{\varepsilon_{0} m^{*}} \frac{1}{\omega^{2}+\mathrm{i} \Gamma_{\mathrm{p}} \omega}+\sum_{j=1}^{4} \frac{S_{j} \omega_{\mathrm{TO}, j}^{2}}{\omega_{\mathrm{TO}, j}^{2}-\omega^{2}-\mathrm{i} \Gamma_{j} \omega} .
$$

The third and fourth terms in (1) represent the free-carrier and phonon contributions described by the classical plasmon and phonon oscillators. In Eq. (1) the following notations are used: $N$ - free carrier concentration, $\varepsilon_{0}$ - permittivity of free space, $m^{*}$ - effective mass of free carriers, $\Gamma_{\mathrm{p}}$ - damping constant of plasma oscillation, $\omega_{\mathrm{TO}, j}, \Gamma_{j}$ are the frequencies and damping constants of phonon oscillators, $S_{j}$ is the strength of the $j$-th phonon oscillator. The summation contains three phonon modes of the three alloy constituents and the additional $\Omega_{2}$ mode. The first and second terms in (1) are the interband contribution to $\varepsilon(\omega): \varepsilon_{\infty}^{\prime}$ is the contribution due to all interband transitions except $\Gamma_{8}^{\mathrm{y}} \rightarrow \Gamma_{8}^{\mathrm{c}}$ (being real and frequency independent) and $\Delta \varepsilon_{\text {inter }}(\omega)$ is the contribution due to $\Gamma_{8}^{\mathrm{y}} \rightarrow \Gamma_{8}^{\mathcal{c}}$ transitions and is described in $[4,6]$. The calculated reflectivity curves fit relatively well to the experimental reflectivity spectra (Fig. 1). The fitting parameters, given in Table, are reasonably comparable, in the sense of virtual crystal approximation with literature data for HgTe and its ternary alloys [4-15].

The quaternary $\mathrm{HgCdMnTe}$ alloy gives the opportunity to compare the strength of phonon modes of the constituents in respect to their molar concentrations. The relatively high strength of MnTe-like mode is noticeable. The $\Omega_{2}$ 
mode is similar to that in $\mathrm{HgTe}$ and $\mathrm{HgTe}$-rich $\mathrm{HgCdTe}$ but slightly broader. Its strength is strongly temperature dependent; at $90 \mathrm{~K}$ it manifests as a bend point. The energy of the longitudinal optical phonon of HgTe-like mode agrees well with the results obtained from phonon assisted interband magnetoabsorption in our previous measurements of $\mathrm{HgTe}$-rich $\mathrm{HgCdTe}$ samples [16].

\section{References}

[1] J. Kossut, in: Semiconductors and Semimetals, Eds. J.K. Furdyna, J. Kossut, Vol. 25, Academic Press, San Diego 1988, p. 183.

[2] N.L. Bazlenov, S.I. Gasanov, V.I. Ivanov-Omski, K.E. Mironov, V.F. Movile, Infrared Phys. 33, 169 (1992).

[3] U. Dębska, M. Dietl, G. Grabecki, E. Janik, E. Kierzek-Pelcod, M. Ḱlimkiewicz, Phys. Status Solidi A 64, 707 (1981).

[4] M. Grynberg, R. Le Toullec, M. Balkanski, Phys. Rev. B 9, 517 (1974).

[5] P. Świątek, A.M. Witowski, M. Grynberg, Phys. Status Solidi B 89, K1 (1978).

[6] A. Polian, R. Le Toullec, M. Balkanski, Phys. Rev. B 13, 3558 (1976).

[7] A.M. Witowski, M. Grynberg, Phys. Status Solidi B 100, 389 (1980).

[8] J. Baars, F. Sorger, Solid State Commun. 10, 875 (1972).

[9] S.W. McKnight, P.M. Amirtharaj, S. Perkowitz, Solid State Commun. 25, 357 (1978).

[10] W. Lu, Z.Y. Yu, H.J. Ye, W.L. Xu, K.J. Ma, S.C. Shen, Phys. Rev. B 40, 3383 (1989).

[11] N.N. Gavaleshko, S.I. Kriven, A.P. Litvinchuk, Yu.I. Mazur, S.Yu. Paranchich, Semicond. Sci. Technol. 5, S307 (1990).

[12] J.H. Chu, S.C. Shen, Semicond. Sci. Technol. 8, S86 (1993).

[13] J.M. Wróbel, B.P. Claiman, P. Becla, R. Sudharsanan, S. Perkowitz, J. Appl. Phys. 64, 310 (1988).

[14] W. Gębicki, W. Nazarewicz, Phys. Status Solidi B 80, 307 (1977).

[15] W. Gębicki, W. Nazarewicz, Phys. Stalus Solidi B 86, K135 (1978).

[16] W. Zawadzki, E. Dudziak, L.Z. Jędral, E. Placzek-Popko, J. Bożym, Semicond. Sci. Technol. 8, S172 (1993). 